\title{
Desempenhos nas Duas Tarefas do Subteste Dígitos do WISC-III e do WAIS-III
}

\author{
Vera L. M. de Figueiredo ${ }^{1}$ \\ Universidade Católica de Pelotas \\ Elizabeth do Nascimento \\ Universidade Federal de Minas Gerais
}

\begin{abstract}
RESUMO - O subteste Dígitos está incluído no WISC-III e no WAIS-III, constituindo uma medida de atenção e memória de trabalho. Considerando que o desempenho em cada parte desse subteste implica em funções cognitivas diferentes, a determinação dos padrões de desempenho e das discrepâncias pode ser útil na investigação clínica. O artigo apresenta os resultados das amostras de normatização brasileiras dos testes WISC-III e WAIS-III nas ordens direta e inversa dos dígitos, analisando-se as discrepâncias entre cada uma delas. Os sujeitos recordaram mais dígitos na ordem direta do que na inversa. No WISC-III, a mediana da quantidade máxima de dígitos que as crianças memorizaram foi de cinco na ordem direta e três na ordem inversa. No WAIS-III, a mediana alcançada pela amostra foi de cinco dígitos na ordem direta e de quatro na inversa. O número de dígitos retido foi menor que o reportado para as amostras de normatização americanas.
\end{abstract}

Palavras-chave: memória de trabalho; atenção; Dígitos; WISC-III; WAIS-III.

\section{Performances in the Forward and Backward Digit Span in the WISC-III and WAIS-III}

\begin{abstract}
Digit Span subtest is comprised in the WISC-III and WAIS-III and it constitutes an attention and working memory measure. Assuming that performance in each part of this subtest implies in different cognitive functions, the determination of performance patterns and of discrepancies can be useful in clinical evaluation. The present article shows the results of the samples of Brazilian standardization of the tests WISC-III and WAIS-III in the forward and backward orders of the digits, analyzing the discrepancies between each of them. Subjects remembered a larger number of digits in the forward than in the backward. In WISC-III, the median of the maximum amount of digits retained by children was five digits in the forward and three in the backward. In WAIS-III, the median was five digits in the forward and four digits in the backward. The number of digits retained was lower than the reported for the American standardization samples.
\end{abstract}

Key words: working memory; attention; Digit Span; WISC-III; WAIS-III.

Entre os subtestes das Escalas Wechsler de Inteligência, Dígitos é um dos mais investigados e utilizados (Prifitera \& Saklofske, 1998; Zimmerman \& Woo-Sam, 1981). Segundo Glasser e Zimmerman (1977), as tarefas de repetição de números sempre foram largamente usadas por psiquiatras e psicólogos como uma prova simples de memória. Segundo dados da literatura, espera-se que adultos com inteligência normal sejam capazes de reter pelo menos cinco dígitos na ordem direta e três na ordem inversa, enquanto uma criança de 7 anos consegue reter, no mínimo, quatro dígitos na ordem direta e dois na inversa. A diferença de um ou dois dígitos é usualmente encontrada em pessoas normais até a meia idade (Cunha, 1993).

Historicamente, a combinação da ordem direta e indireta no subteste Dígitos se deu por questões práticas, pois a consideração de cada uma isoladamente limitaria os resultados brutos e daria grande ênfase à memória de curto prazo na determinação do QI (Kaufman, 1990; Tulsky \& cols., 2003). No entanto, Kaufman (1990), Lezak (1995)

1 Endereço: Rua Quinze de Novembro, 457/704, Pelotas, RS, Brasil 96015-000. E-mail: verafig@ @erra.com.br e Reynolds (1997) estão entre os autores que consideram que a combinação das ordens direta e inversa em um único escore reduz o valor clínico da tarefa.

Diferenças entre os desempenhos na ordem direta e na inversa do subteste Dígitos têm sido amplamente investigadas (Hale, Hoeppner \& Fiorello, 2002; Kaufman, 1994; Reynolds, 1997; Rosenthal, Riccio, Gsanger \& Jarratt, 2006; Wechsler, 1991). Entre os trabalhos em que houve um melhor desempenho na ordem direta, Wechsler (1991) cita os estudos de Rudel e Denkla, no qual as crianças com perturbações no desenvolvimento que resultaram em prejuízo no hemisfério direito mostraram melhor desempenho na execução dessa tarefa. Para Kaufman (1994), indivíduos com danos no hemisfério direito apresentam a impossibilidade de realizar a tarefa de repetir dígitos em ordem inversa porque esta se encontra relacionada com habilidade viso-espacial do cérebro. Segundo Zimmerman e Woo-Sam (1981, pp.126-127), a discrepância entre as pontuações a favor da ordem direta "pode ser devida a escassa memória auditiva, ansiedade, a uma simples falta de atenção, ou a pouca capacidade mental'. Kaufman (1990) e Lezak (1995) afirmam que grandes diferenças (cinco ou mais dígitos) em favor da ordem direta ocorrem raramente 
em pessoas normais, mas são mais comuns em pessoas com danos cerebrais.

Para Glasser e Zimmerman (1977) e outros clínicos como Kitzinger e Blumberg, (citados por Kaufman, 1994), a capacidade de repetir mais dígitos na ordem inversa pode indicar obstinação quando a tarefa de repetir a ordem direta é considerada demasiadamente fácil e por isso é rechaçada. Nesse caso, pode-se tratar de indivíduos com motivação elevada para as atividades intelectuais, uma indicação de controle e flexibilidade mental ou, ainda, um bom nível de tolerância à tensão.

Atualmente, predomina na literatura a consideração de que o subteste Dígitos avalia principalmente a capacidade de memória de trabalho (auditiva) e a atenção (Kaufman; 1994; Kaufman \& Lichtenberger, 1999), e que as duas tarefas que o compõe requerem processamentos cognitivos distintos (Gregory, 1999; Kaufman \& Lichtenberger, 1999; Reynolds, 1997; Tulsky \& cols., 2003). O modelo proposto em 1974 por Baddeley e Hitch (citado por Eysenck \& Keane, 1994) acerca da memória de trabalho, tem se constituído em uma referência importante para auxiliar na compreensão das discrepâncias nos desempenhos nesse tipo de tarefa (Rosenthal $\&$ cols., 2006). Esse modelo compreende três componentes (um executivo central e dois outros sistemas denominados circuito fonológico e tábua de rascunho viso-espacial) que auxiliam na manutenção temporária e no processamento da informação.

Com base nesse modelo, embora as duas tarefas do subteste Dígitos envolvam a repetição de números apresentados oralmente pelo examinador, a tarefa solicitada na ordem inversa apresenta nitidamente maior grau de complexidade, estando, portanto, relacionada com o executivo central. Esse sistema de controle atencional seria responsável por estratégias de seleção, controle e coordenação dos vários processos envolvidos na armazenagem de curto prazo, exigindo, simultaneamente, a armazenagem e o processamento da informação. A tarefa solicitada na ordem direta estaria relacionada particularmente com o circuito fonológico, uma vez que a sua realização envolve apenas a armazenagem passiva e temporária de material baseado na fala, com pouca demanda do sistema do executivo central.

Assim, com base no modelo supracitado, cada tarefa do subteste Dígitos envolve processos cognitivos diferentes. Como a ordem inversa demanda mais do sistema executivo central e da atenção, em razão de sua maior complexidade quando comparada com a ordem direta, torna-se, portanto, compreensível a observação de uma tendência geral de melhor desempenho na ordem direta do que na inversa.

Considerando o exposto, parece ser bastante útil, para uma avaliação clínica, considerar, separadamente, os desempenhos em cada uma das tarefas de Dígitos para levantamento de hipóteses diagnósticas (Kaufman, 1990; Lezak, 1995; Prifitera \& Saklofske, 1998; Sattler, 1988; Tulsky \& cols., 2003; Wechsler, 1991).

Para analisar as discrepâncias observadas nos desempenhos entre a ordem direta e a inversa, Kaplan (citado por The Psychological Corporation, 1997) desenvolveu dois procedimentos. O primeiro consiste em comparar os resultados individuais com o desempenho da amostra de normatização quanto ao maior número de dígitos lembrados na ordem direta e na inversa. Como observa Kaufman (1994), nesse procedimento o importante é identificar qual o maior número de dígitos que o examinando foi capaz de repetir, não importando se ele acertou uma tentativa ou as duas tentativas de uma determinada série. Essa série máxima, entretanto, não corresponde ao número de pontos (escore bruto) que o examinando obteve em cada parte do subteste.

O segundo procedimento consiste em calcular a diferença entre os desempenhos máximos nas duas ordens e compará-lo com a amostra normativa. Os dois processos podem auxiliar o examinador na determinação, por exemplo, de déficits de atenção, que se tornam mais aparentes quando as duas tarefas do subteste são avaliadas separadamente (Hale \& cols., 2002). Kaufman (1994) sugere, também, comparar os resultados individuais do menor número de dígitos lembrados em cada uma das ordens com o desempenho da amostra de normatização.

Os manuais americanos de aplicação e correção do WISCIII e WAIS-III não oferecem escores ponderados separados para cada ordem. No entanto, trazem tabelas que apresentam o desempenho da amostra de normatização com o objetivo de oferecer os parâmetros para análise das discrepâncias entre os resultados individuais e o da população (Tabelas B6 dos manuais Wechsler, 1991; Wechsler, 1997).

Em relação aos parâmetros encontrados na amostra de padronização norte-americana do WISC-III (Wechsler, 1991), a mediana encontrada para a amostra geral na ordem direta foi de seis dígitos e para a ordem inversa quatro dígitos. No WAIS-III, os parâmetros encontrados na amostra de padronização norte-americana (Wechsler, 1997) revelam que na amostra total e em todos os grupos etários houve a recordação de mais números na ordem direta do que na inversa, sendo que para a amostra geral a diferença na mediana foi de dois pontos a favor da primeira ordem. Ao se considerar a amostra total de normatização, a mediana na ordem direta foi de seis dígitos e na ordem inversa de cinco dígitos. Sattler e Ryan (1999) destacam que apenas 3,8\% da amostra total de normatização americana recordaram maior número de dígitos na ordem inversa do que na ordem direta.

Considerando a relevância das informações sobre o desempenho das amostras de padronização enquanto parâmetros para análise das discrepâncias nos desempenhos em cada uma das partes do subteste Dígitos, o presente estudo apresenta dados relativos às discrepâncias observadas no subteste Dígitos pelas amostras de normatização do WISC-III e do WAIS-III para uso no Brasil. Os dados poderão auxiliar os profissionais na análise dos perfis individuais de crianças e adultos, principalmente de grupos clínicos.

\section{Método}

\section{Amostra}

Os dados aqui analisados pertencem aos estudos de adaptação, validação e normatização brasileira do WISC-III (Figueiredo, 2001) e do WAIS-III (Nascimento, 2000).

A amostra do WISC-III $(N=801)$ foi constituída por crianças residentes na região sul do Brasil, representando em torno de $1 \%$ da população geral de escolares da cidade de Pelotas/RS. As idades variaram de 6 até 16 anos, com 
proporção similar de meninos (49\%) e meninas (51\%). As crianças selecionadas estavam matriculadas em 34 instituições de ensinos público e privado, localizadas tanto no centro como na periferia. As escolas foram escolhidas pelo critério de abrangência de ensino (ter, prioritariamente, primeiro e segundo graus) e localização (diferentes bairros da zona urbana da cidade). A seleção das crianças nas escolas foi realizada com base na listagem dos alunos por série, tentando-se escolher ao acaso. Para as análises, as idades foram agrupadas em seis faixas etárias, contendo cada grupo em torno de 130 crianças.

A amostra de normatização do WAIS-III $(N=788)$, constituída de pessoas com idades entre 16 e 89 anos, foi delineada de forma a ser a mais representativa possível da população de Belo Horizonte/Minas Gerais. Para tal, foram consideradas as seguintes variáveis para sua composição: idade, sexo e anos de estudo. Participaram do estudo pessoas saudáveis que foram contatadas ora por meio de instituições públicas e privadas ora pela rede social dos pesquisadores. Nessa perspectiva, a amostra foi de conveniência. Foram estabelecidos oito grupos etários e o número de pessoas em cada grupo etário variou entre 83 e 118.

\section{Instrumento}

O subteste Dígitos no WISC-III está incluído entre os subtestes suplementares e, juntamente com a Aritmética, auxilia na medida do Índice de Resistência à Distração. $\mathrm{O}$ WAIS-III é considerado um subteste regular e, juntamente com a Sequiência de Números e Letras e Aritmética, permite o cálculo do Índice de Memória de Trabalho.

Tanto no WISC-III quanto no WAIS-III, o subteste Dígitos é composto de oito séries para ordem direta e sete para inversa, havendo um aumento gradual da quantidade de dígitos em cada série. A ordem direta é aplicada em primeiro lugar, seguida pela inversa, que é administrada independentemente se o examinando fracassa totalmente na ordem direta. Cada item é formado de dois conjuntos de dígitos constituindo em duas tentativas, sendo ambas aplicadas. A pontuação máxima no subteste é de 30 pontos, sendo que o resultado bruto máximo na ordem direta é de 16 pontos enquanto na ordem inversa é de 14 pontos.

\section{Procedimentos}

Os dados sobre os desempenhos das amostras em estudo no subteste Dígitos foram coletados durante a aplicação dos testes WISC-III e WAIS-III.

Operacionalmente, a tarefa exigida no subteste Dígitos consiste em apresentar oralmente séries de dígitos para o examinando repetir na mesma ordem em que são lidos pelo examinador (ordem direta) e outros em ordem contrária (ordem inversa). Tanto na ordem direta quanto na inversa, a aplicação é suspensa após o fracasso nas duas tentativas do mesmo item. O subteste Dígitos não apresenta tempo limite. Conforme orientações contidas nos manuais dos testes WISC-III e WAIS-III, as respostas dos examinandos no subteste foram registradas pelo examinador.

Os procedimentos de análises dos dados incluíram: 1) o cálculo das medianas e das médias dos escores brutos para levantamento dos padrões de desempenhos das amostras normativas em função da idade; 2) em cada ordem, foi identificado o número máximo de dígitos memorizados por cada um dos sujeitos de ambas as amostras, conforme procedimento citado por Kaplan, Fein, Morris e Delis (1991), e posteriormente as respectivas porcentagens observadas; 3) as discrepâncias entre o desempenho na ordem direta e inversa foram calculadas pela diferença encontrada entre $o$ número máximo de dígitos repetido corretamente em cada ordem e 4) ao analisar as discrepâncias era importante, também, observar se elas são usuais ou incomuns. Por essa razão, tornou-se relevante analisar com que freqüência a diferença observada ocorreu na amostra de padronização.

\section{Resultados}

Na Tabela 1 observa-se que no WISC-III, tanto na ordem direta quanto inversa, houve um aumento progressivo dos escores médios ao longo das faixas etárias. Na ordem direta, o escore bruto mediano da amostra total no WISC-III foi de sete e na ordem inversa foi de quatro. A diferença entre as medianas foi de três pontos a favor da ordem direta, com exceção das faixas etárias de sete anos e 14-16 anos, em que a diferença foi de quatro e dois, respectivamente.

Observa-se que na ordem direta a amostra total no WAIS-III apresentou um resultado bruto mediano de sete dígitos, enquanto na ordem inversa apresentou um resultado de cinco. A diferença entre as medianas na ordem direta e inversa foi de dois pontos a favor da primeira, com exceção do grupo etário 30-39 anos, no qual foi de três pontos.

Conforme o primeiro procedimento proposto por Kaplan e colaboradores (1991), a Tabela 2 apresenta as porcentagens de sujeitos para o número máximo de dígitos alcançados nas ordens direta e inversa. Em todas as faixas etárias, tanto entre as crianças quanto entre os adultos, houve maior memorização de números na ordem direta do que na inversa.

Considerando o segundo procedimento proposto por Kaplan e colaboradores, as Tabelas 3 e 4 apresentam as porcentagens da diferença entre os maiores desempenhos alcançados na ordem direta e inversa, por grupo etário. Verifica-se que em todas as faixas etárias houve uma porcentagem nula ou pequena de sujeitos que acertaram mais itens na ordem inversa que na direta (valores negativos).

Em relação à Tabela 3, observa-se que no WISC-III a diferença de até um dígito a favor da ordem inversa ocorreu nas seguintes idades: 6 anos, 7 anos e 8-9 anos. A diferença de dois dígitos a mais na ordem inversa do que na direta ocorreu apenas entre as crianças maiores (na faixa etária a partir de 12 anos).

Na Tabela 4 verifica-se que, no WAIS-III, cinco grupos etários apresentaram uma discrepância de até um dígito a mais na ordem inversa do que na direta. Dois grupos etários apresentaram uma discrepância de até dois dígitos a favor da ordem inversa (16-17 e 40-49 anos). Apenas o grupo etário 20-29 anos apresentou uma discrepância de até três dígitos a mais na ordem inversa do que na direta.

As Tabelas 5 e 6 foram elaboradas para facilitar a interpretação das frequiências das discrepâncias. Nelas são apresentadas as quantidades maiores e menores de dígitos 
Tabela 1. Estatísticas dos escores brutos nas ordens direta e inversa do subteste Dígitos no WISC-III e WAIS-III adaptados, por grupo etário.

\begin{tabular}{|c|c|c|c|c|c|c|c|c|c|c|c|c|c|c|c|c|c|c|}
\hline \multicolumn{19}{|c|}{ Grupos etários } \\
\hline \multirow[t]{2}{*}{ WISC-III } & \multicolumn{2}{|c|}{6} & \multicolumn{2}{|c|}{7} & \multicolumn{2}{|c|}{ 8-9 } & \multicolumn{2}{|c|}{$10-11$} & \multicolumn{2}{|c|}{$12-13$} & \multicolumn{2}{|c|}{$14-16$} & & & & & \multicolumn{2}{|c|}{ Todas idades } \\
\hline & D & I & D & I & D & I & D & I & D & I & D & I & & & & & D & I \\
\hline Média & 5,27 & 1,68 & 5,72 & 2,27 & 6,36 & 3,26 & 7,55 & 4,16 & 7,84 & 4,94 & 8,59 & 5,67 & & & & & 6,88 & 3,66 \\
\hline DP & 1,62 & 1,39 & 1,84 & 1,34 & 1,46 & 1,26 & 1,77 & 1,62 & 1,82 & 1,77 & 1,99 & 1,97 & & & & & 2,11 & 2,11 \\
\hline Mediana & 5,00 & 2,00 & 6,00 & 2,00 & 6,00 & 3,00 & 7,00 & 4,00 & 8,00 & 5,00 & 8,00 & 6,00 & & & & & 7,00 & 4,00 \\
\hline Amplitude & $1 / 14$ & $0 / 5$ & $2 / 12$ & $0 / 6$ & $2 / 11$ & $0 / 7$ & $4 / 12$ & $1 / 11$ & $4 / 14$ & $2 / 10$ & $5 / 14$ & $2 / 13$ & & & & & $1 / 14$ & $0 / 13$ \\
\hline \multicolumn{19}{|l|}{ Diferença D-I } \\
\hline Média (DP) & $\begin{array}{c}3,59 \\
(1,68)\end{array}$ & & $\begin{array}{c}3,45 \\
(1,87)\end{array}$ & & $\begin{array}{c}3,09 \\
(1,69)\end{array}$ & & $\begin{array}{c}3,39 \\
(1,81)\end{array}$ & & $\begin{array}{c}2,90 \\
(1,92)\end{array}$ & & $\begin{array}{c}2,91 \\
(2,00)\end{array}$ & & & & & & $\begin{array}{c}3,22 \\
(1,84)\end{array}$ & \\
\hline $\mathrm{N}$ & 137 & & 128 & & 137 & & 133 & & 137 & & 129 & & & & & & 801 & \\
\hline \multicolumn{19}{|c|}{ Grupos etários } \\
\hline \multirow[t]{2}{*}{ WAIS-III } & \multicolumn{2}{|c|}{$16-17$} & \multicolumn{2}{|c|}{$18-19$} & \multicolumn{2}{|c|}{$20-29$} & \multicolumn{2}{|c|}{ 30-39 } & \multicolumn{2}{|c|}{$40-49$} & \multicolumn{2}{|c|}{$50-59$} & \multicolumn{2}{|c|}{$60-64$} & \multicolumn{2}{|c|}{$65-89$} & \multicolumn{2}{|c|}{ Todas idades } \\
\hline & D & I & D & I & D & I & D & I & D & I & D & I & D & I & D & I & D & I \\
\hline Média & 7,46 & 5,30 & 7,27 & 5,34 & 7,30 & 5,67 & 7,10 & 4,69 & 6,72 & 4,56 & 6,84 & 4,59 & 6,59 & 4,35 & 6,10 & 3,76 & 6,93 & 4,81 \\
\hline $\mathrm{DP}$ & 1,96 & 2,16 & 2,26 & 2,46 & 2,38 & 2,69 & 2,25 & 2,21 & 1,92 & 2,19 & 2,60 & 2,31 & 2,14 & 2,00 & 1,89 & 1,84 & 2,22 & 2,35 \\
\hline Mediana & 7,00 & 5,00 & 7,00 & 5,00 & 7,00 & 5,00 & 7,00 & 4,00 & 6,00 & 4,00 & 6,00 & 4,00 & 6,00 & 4,00 & 6,00 & 4,00 & 7,00 & 5,00 \\
\hline Amplitude & $4 / 12$ & $0 / 10$ & $3 / 14$ & $0 / 14$ & $4 / 15$ & $1 / 12$ & $2 / 14$ & $0 / 10$ & $4 / 13$ & $1 / 10$ & $0 / 15$ & $0 / 12$ & $3 / 13$ & $0 / 9$ & $1 / 11$ & $0 / 8$ & $0 / 15$ & $0 / 14$ \\
\hline \multicolumn{19}{|l|}{ Diferença D-I } \\
\hline Média (DP) & $\begin{array}{c}2,16 \\
(2,02)\end{array}$ & & $\begin{array}{c}1,93 \\
(1,98)\end{array}$ & & $\begin{array}{c}1,63 \\
(2,10)\end{array}$ & & $\begin{array}{c}2,37 \\
(1,88)\end{array}$ & & $\begin{array}{c}2,17 \\
(1,82)\end{array}$ & & $\begin{array}{c}2,25 \\
(1,82)\end{array}$ & & $\begin{array}{c}2,24 \\
(1,79)\end{array}$ & & $\begin{array}{c}2,34 \\
(1,79)\end{array}$ & & $\begin{array}{c}2,12 \\
(1,92)\end{array}$ & \\
\hline $\mathrm{N}$ & 93 & & 89 & & 118 & & 109 & & 109 & & 96 & & 83 & & 91 & & 788 & \\
\hline
\end{tabular}

Nota $\mathrm{D}=$ Ordem Direta; $\mathrm{I}=$ Ordem Inversa.

Tabela 2. Percentagens da quantidade máxima de dígitos memorizados na ordem direta e inversa, por grupo etário, no WISC-III e WAIS-III adaptados.

\begin{tabular}{|c|c|c|c|c|c|c|c|c|c|c|c|c|c|c|c|c|c|c|}
\hline \multirow{3}{*}{$\begin{array}{l}\text { WISC-III } \\
\text { Dígitos }\end{array}$} & & & \multicolumn{16}{|c|}{ Grupos etários } \\
\hline & \multicolumn{2}{|c|}{6} & \multicolumn{2}{|c|}{7} & \multicolumn{2}{|c|}{$8-9$} & \multicolumn{2}{|c|}{$10-11$} & \multicolumn{2}{|c|}{$12-13$} & \multicolumn{2}{|c|}{$14-16$} & & & & & \multicolumn{2}{|c|}{ Todas idades } \\
\hline & $\mathrm{D}$ & I & $\mathrm{D}$ & I & $\mathrm{D}$ & I & $\mathrm{D}$ & I & $\mathrm{D}$ & I & $\mathrm{D}$ & I & & & & & $\mathrm{D}$ & I \\
\hline 9 & 0,0 & & 0,8 & & & & 0,0 & & 0,0 & & 1,6 & & & & & & 0,4 & \\
\hline 8 & 0,7 & & 0,0 & & 0,0 & & 3,0 & 0,0 & 3,6 & 0,7 & 10,0 & 1,6 & & & & & 2,8 & 0,4 \\
\hline 7 & 0,0 & & 2,3 & & 2,9 & & 12,8 & 0,8 & 13,2 & 1,5 & 14,8 & 3,8 & & & & & 7,7 & 1,0 \\
\hline 6 & 1,5 & & 6,3 & 0,0 & 5,1 & 0,0 & 11,3 & 2,2 & 17,5 & 5,1 & 21,7 & 3,9 & & & & & 10,4 & 1,8 \\
\hline 5 & 19,0 & 0,0 & 26,5 & 0,8 & 38,7 & 2,9 & 50,3 & 9,0 & 44,5 & 16,5 & 44,1 & 28,7 & & & & & 37,3 & 9,8 \\
\hline 4 & 53,3 & 2,2 & 39,1 & 3,9 & 43,8 & 19,0 & 21,1 & 22,6 & 20,5 & 36,8 & 7,8 & 40,3 & & & & & 31,0 & 20,6 \\
\hline 3 & 20,4 & 26,3 & 23,4 & 36,7 & 8,8 & 50,4 & 1,5 & 55,6 & 0,7 & 35,0 & & 20,9 & & & & & 9,2 & 37,6 \\
\hline 2 & 5,1 & 39,4 & 1,6 & 45,3 & 0,7 & 25,5 & & 9,8 & & 4,4 & & 0,8 & & & & & 1,2 & 20,8 \\
\hline 0 & & 32,1 & & 13,3 & & 2,2 & & & & & & & & & & & & 8,0 \\
\hline Média & 3,94 & 1,66 & 4,23 & 2,20 & 4,47 & 2,93 & 5,22 & 3,41 & 5,33 & 3,91 & 5,8 & 4,32 & & & & & $4, .83$ & 3,07 \\
\hline DP & 0,88 & 1,24 & 1,08 & 1,05 & 0,87 & 0,87 & 1,06 & 0,92 & 1,08 & 1,07 & 1,19 & 1,09 & & & & & 1,22 & 1,39 \\
\hline Mediana & 4,00 & 2,00 & 4,00 & 2,00 & 4,00 & 3,00 & 5,00 & 3,00 & 5,00 & 4,00 & 5,00 & 4,00 & & & & & 5,00 & 3,00 \\
\hline $\mathrm{N}$ & \multicolumn{2}{|c|}{137} & \multicolumn{2}{|c|}{128} & \multicolumn{2}{|c|}{137} & \multicolumn{2}{|c|}{133} & \multicolumn{4}{|c|}{129} & & & & & \multicolumn{2}{|c|}{801} \\
\hline WAIS-III & & & \multicolumn{16}{|c|}{ Grupos Etários } \\
\hline \multirow[t]{2}{*}{ Dígitos } & \multicolumn{2}{|c|}{$16-17$} & \multicolumn{2}{|c|}{ 18-19 } & \multicolumn{2}{|c|}{ 20-29 } & \multicolumn{2}{|c|}{$30-39$} & \multicolumn{2}{|c|}{$40-49$} & \multicolumn{2}{|c|}{$50-59$} & \multicolumn{2}{|c|}{$60-64$} & & & Todas & idades \\
\hline & $\mathrm{D}$ & I & $\mathrm{D}$ & I & D & I & $\mathbf{D}$ & I & $\mathbf{D}$ & I & D & I & D & I & D & I & $\mathrm{D}$ & I \\
\hline 9 & 1,1 & & 0,0 & & 1,7 & & 0,9 & & 0,0 & & 2,1 & & 0,0 & & & & 0,8 & \\
\hline 8 & 1,1 & 0,0 & 5,6 & 2,2 & 5,1 & 3,4 & 2,8 & 1,8 & 1,8 & 0,0 & 1,0 & 1,0 & 2,4 & & 0,0 & & 2,5 & 1,1 \\
\hline 7 & 13,9 & 4,3 & 12,4 & 1,2 & 9,3 & 6,8 & 11,0 & 1,0 & 6,5 & 1,8 & 11,5 & 2,1 & 3,6 & 0,0 & 5,5 & 0,0 & 9,3 & 2,3 \\
\hline 6 & 23,7 & 6,5 & 13,5 & 10,1 & 16,1 & 13,5 & 15,6 & 7,3 & 13,7 & 6,5 & 17,7 & 3,2 & 24,1 & 3,6 & 16,5 & 2,2 & 17,3 & 6,9 \\
\hline 5 & 29,0 & 21,5 & 29,2 & 21,3 & 29,7 & 13,6 & 26,6 & 11,0 & 32,1 & 15,6 & 16,7 & 17,7 & 20,5 & 18,1 & 15,4 & 8,8 & 25,3 & 15,7 \\
\hline 4 & 30,9 & 33,3 & 28,1 & 30,4 & 30,5 & 28,8 & 36,7 & 31,2 & 35,8 & 27,5 & 40,6 & 28,1 & 33,7 & 30,1 & 45,0 & 25,3 & 34,6 & 29,3 \\
\hline 3 & 0,3 & 22,6 & 11,2 & 22,4 & 7,6 & 22,9 & 5,5 & 33,0 & 10,1 & 27,5 & 8,3 & 32,3 & 15,7 & 30,1 & 16,5 & 37,3 & 9,7 & 28,5 \\
\hline 2 & & 10,7 & & 11,3 & & 11,0 & 0,9 & 11,9 & & 21,1 & 1,1 & 10,4 & & 14,5 & 1,1 & 20,9 & 0,4 & 13,9 \\
\hline 0 & & 1,1 & & 1,1 & & & & 2,8 & & & 1,0 & 5,2 & & 3,61 & & 5,5 & 0,1 & 2,3 \\
\hline Média & 5,24 & 3,99 & 5,04 & 4,04 & 5,11 & 4,30 & 4,99 & 3,68 & 4,76 & 3,64 & 4,86 & 3,60 & 4,73 & 3,52 & 4,46 & 3,12 & 4,91 & 3,75 \\
\hline DP & 1,21 & 1,31 & 1,37 & 1,40 & 1,37 & 1,56 & 1,29 & 1,39 & 1,13 & 1,26 & 1,47 & 1,45 & 1,23 & 1,25 & 1,15 & 1,23 & 1,30 & 1,40 \\
\hline Mediana & 5,00 & 4,00 & 5,00 & 4,00 & 5,00 & 4,00 & 5,00 & 4,00 & 5,00 & 4,00 & 4,00 & 4,00 & 5,00 & 4,00 & 4,00 & 3,00 & 5,00 & 4,00 \\
\hline $\mathrm{N}$ & & & & & & & & & & & & & 8 & & 9 & & & \\
\hline
\end{tabular}

Nota. $\mathrm{D}=$ Ordem Direta; $\mathrm{I}$ = Ordem Inversa. 
Tabela 3. WISC-III: percentagens da diferença entre o máximo de dígitos memorizados na ordem direta e ordem inversa, por grupo etário.

\begin{tabular}{lccccccc}
\hline \multirow{2}{*}{$\begin{array}{l}\text { Direta } \\
\text { menos }\end{array}$} & \multicolumn{7}{c}{ Grupos etários } \\
\cline { 2 - 8 } $\begin{array}{l}\text { Inversa } \\
7\end{array}$ & 6 & 7 & $8-9$ & $10-11$ & $12-13$ & $14-16$ & $\begin{array}{c}\text { Todas } \\
\text { idades }\end{array}$ \\
\cline { 2 - 8 } 7 & & 0,8 & & & & & 0,1 \\
6 & 0,0 & 0,0 & 0,0 & 0,0 & 0,0 & 0,0 & 0,0 \\
5 & 3,6 & 2,3 & 1,5 & 3,0 & 0,7 & 0,8 & 2,0 \\
4 & 14,6 & 10,2 & 3,6 & 3,0 & 5,9 & 7,0 & 7,4 \\
3 & 19,8 & 20,3 & 11,0 & 14,3 & 12,4 & 12,4 & 15,0 \\
2 & 36,5 & 27,3 & 32,8 & 42,1 & 21,9 & 24,8 & 30,9 \\
1 & 19,7 & 30,5 & 35,8 & 27,1 & 39,4 & 34,1 & 31,1 \\
0 & 5,1 & 7,8 & 13,8 & 10,5 & 15,3 & 17,0 & 11,6 \\
-1 & 0,7 & 0,8 & 1,5 & & 2,9 & 3,1 & 1,5 \\
-2 & & & & & 1,5 & 0,8 & 0,4 \\
Média & 2,28 & 2,03 & 1,55 & 1,81 & 1,42 & 1,48 & 1,76 \\
DP & 1,23 & 1,31 & 1,11 & 1,10 & 1,26 & 1,28 & 1,25 \\
Mediana & 2,00 & 2,00 & 1,00 & 2,00 & 1,00 & 1,00 & 2,00 \\
$\mathrm{~N}$ & 137 & 128 & 137 & 133 & 137 & 129 & 801 \\
\hline
\end{tabular}

Tabela 4. WAIS-III: percentagens da diferença entre o máximo de dígitos memorizados na ordem direta e ordem inversa, por grupo etário.

\begin{tabular}{lccccccccc}
\hline \multirow{2}{*}{$\begin{array}{l}\text { Direta } \\
\text { menos }\end{array}$} & \multicolumn{10}{c}{ Grupos etários } \\
\cline { 2 - 10 } Inversa & $16-17$ & $18-19$ & $20-29$ & $30-39$ & $40-49$ & $50-59$ & $60-64$ & $65-89$ & $\begin{array}{c}\text { Todas } \\
\text { idades }\end{array}$ \\
\cline { 2 - 10 } 5 & 1,1 & 0,0 & & 0,9 & 0,0 & 0,0 & 1,2 & 0,0 & 0,4 \\
4 & 2,1 & 2,2 & 0,0 & 0,9 & 0,9 & 3,1 & 1,2 & 5,5 & 1,9 \\
3 & 14,0 & 9,0 & 7,6 & 12,0 & 12,9 & 10,4 & 10,9 & 9,9 & 10,8 \\
2 & 23,7 & 20,9 & 28,0 & 29,3 & 22,0 & 24,0 & 28,9 & 29,7 & 25,9 \\
1 & 31,1 & 34,2 & 26,3 & 34,9 & 33,0 & 40,6 & 26,5 & 28,5 & 31,8 \\
0 & 19,4 & 21,3 & 20,3 & 15,6 & 24,8 & 15,7 & 25,3 & 20,9 & 20,3 \\
-1 & 7,5 & 12,4 & 12,7 & 6,4 & 5,5 & 6,2 & 6,0 & 5,5 & 7,9 \\
-2 & 1,1 & & 4,3 & & 0,9 & & & & 0,9 \\
-3 & & & 0,8 & & & & & & 0,1 \\
Média & 1,25 & 1,0 & 0,81 & 1,31 & 1,12 & 1,26 & 1,22 & 1,34 & 1,15 \\
DP & 1,31 & 1,22 & 1,34 & 1,15 & 1,17 & 1,14 & 1,22 & 1,23 & 1,23 \\
Mediana & 1,0 & 1,0 & 1,0 & 1,0 & 1,0 & 1,0 & 1,0 & 1,0 & 1,0 \\
$\mathrm{~N}$ & 93 & 89 & 118 & 109 & 109 & 96 & 83 & 91 & 788 \\
\hline & & & & & & & & & \\
\hline
\end{tabular}

alcançados por cada faixa etária, tanto no WISC-III quanto no WAIS-III. As frequiências de $1 \%, 5 \%$ e $15 \%$ indicam resultados incomuns e, quando encontrados no protocolo de um examinando, devem ser investigados. Para Kaufman (1994), a discrepância que se observa em até $15 \%$ dos indivíduos da amostra de normatização é considerada suficientemente rara de ocorrer.

Supondo-se, por exemplo, que uma criança de 7 anos teve êxito na série de seis dígitos na ordem direta e quatro na ordem inversa, a interpretação seria a seguinte: entrando-se na Tabela 5 na parte apropriada (WISC-III/7 anos), observa-se que a retenção de seis dígitos não é incomum; sete dígitos são necessários para ser considerado pouco freqüente para a idade de 7 anos. Como mostra a Tabela 5, a retenção de sete dígitos na ordem direta ocorreu em 5\% das crianças dessa faixa etária e nove dígitos ou mais no extremo de $1 \%$ da amostra. Dessa forma, o desempenho de seis dígitos foi comum de ser memorizado, tendo ocorrido em mais de $15 \%$ da amostra das crianças brasileiras de 7 anos. $\mathrm{O}$ resultado de quatro dígitos na ordem inversa, entretanto, é incomum para essa idade. Segundo a Tabela 5 , a recordação máxima de quatro dígitos ocorreu em somente 5\% das crianças dessa idade. A baixa ocorrência sugere que o examinando pode ter uma boa facilidade para números se outras informações apoiarem essa hipótese.
A Tabela 6 pode ser utilizada da mesma maneira para determinar se o baixo escore do indivíduo na ordem direta ou inversa foi incomum na amostra de padronização.

Tabela 5. Série máxima incomum de dígitos alcançada na ordem direta e inversa, por grupo etário.

\begin{tabular}{|c|c|c|c|c|c|c|c|}
\hline & & \multicolumn{6}{|c|}{ Percentagem da População normal } \\
\hline & & \multicolumn{2}{|c|}{ Até $1 \%$} & \multicolumn{2}{|c|}{ Até $5 \%$} & \multicolumn{2}{|c|}{ Até $15 \%$} \\
\hline & Idade & $\mathrm{D}$ & $\mathrm{I}$ & $\mathrm{D}$ & $\mathrm{I}$ & $\mathrm{D}$ & $\mathrm{I}$ \\
\hline \multirow{6}{*}{ WISC-III } & 6 & 8 & 5 & 6 & 4 & & \\
\hline & 7 & 9 & 5 & 7 & 4 & & \\
\hline & $8-9$ & 8 & 6 & 7 & 5 & 6 & \\
\hline & $10-11$ & 9 & 7 & 8 & 6 & 6 & 5 \\
\hline & $12-13$ & 9 & 8 & 8 & 7 & 7 & 6 \\
\hline & $14-16$ & 9 & 8 & 9 & 7 & 7 & 6 \\
\hline \multirow{8}{*}{ WAIS-III } & $16-17$ & 9 & 8 & 8 & 7 & 7 & 6 \\
\hline & $18-19$ & 9 & & 8 & 8 & & 6 \\
\hline & $20-29$ & & & 9 & 8 & 8 & 7 \\
\hline & $30-39$ & 9 & & 8 & 7 & 7 & 6 \\
\hline & $40-49$ & 9 & 8 & 8 & 7 & 7 & 6 \\
\hline & $50-59$ & & 8 & 9 & 7 & 7 & 6 \\
\hline & $60-64$ & 9 & 7 & 8 & 6 & 7 & \\
\hline & $65-89$ & 8 & 7 & 7 & 6 & & 5 \\
\hline
\end{tabular}

Nota. $\mathrm{D}=$ Ordem Direta; $\mathrm{I}=$ Ordem Inversa.

Tabela 6. Série mínima incomum de dígitos alcançada na ordem direta e inversa, por grupo etário.

\begin{tabular}{|c|c|c|c|c|c|c|c|}
\hline & & \multicolumn{6}{|c|}{ Percentagem da População normal } \\
\hline & & \multicolumn{2}{|c|}{ Até $1 \%$} & \multicolumn{2}{|c|}{ Até $5 \%$} & \multicolumn{2}{|c|}{ Até $15 \%$} \\
\hline & Idade & $\mathrm{D}$ & $\mathrm{I}$ & $\mathrm{D}$ & I & $\mathrm{D}$ & I \\
\hline \multirow{6}{*}{ WISC-III } & 6 & & & & & 2 & \\
\hline & 7 & & & 2 & & & 0 \\
\hline & $8-9$ & 2 & 0 & & 0 & 3 & \\
\hline & $10-11$ & & & 3 & & & 2 \\
\hline & $12-13$ & 3 & & & 2 & & \\
\hline & $14-16$ & & 2 & & & 4 & \\
\hline \multirow{8}{*}{ WAIS-III } & $16-17$ & 3 & & & 0 & & 2 \\
\hline & $18-19$ & & & & 0 & 3 & 2 \\
\hline & $20-29$ & & & & & 3 & 2 \\
\hline & $30-39$ & 2 & & 3 & 0 & & 2 \\
\hline & $40-49$ & & & & & 3 & 0 \\
\hline & $50-59$ & 0 & & 2 & 0 & 3 & 2 \\
\hline & $60-64$ & & & & 0 & 3 & 2 \\
\hline & $65-89$ & & & 2 & 0 & & \\
\hline
\end{tabular}

Nota. $\mathrm{D}=$ Ordem Direta; $\mathrm{I}=$ Ordem Inversa.

\section{Discussão}

Os pontos de referência encontrados na amostra de padronização do WISC-III foram semelhantes aos encontrados na amostra original do teste evidenciando-se, entretanto, uma diferença de menos um dígito no desempenho das crianças brasileiras. A mediana do número máximo de dígitos memorizados pela amostra geral das crianças brasileiras foi de cinco na ordem direta e três na ordem inversa, enquanto na amostra americana foi de seis e quatro, respectivamente. Ao serem comparados os desempenhos das duas amostras no subteste Dígitos, observa-se sempre a diferença de um dígito a favor das crianças americanas.

Quanto à diferença entre o máximo de dígitos memorizados na ordem direta e inversa no WISC-III, tanto na amostra brasileira (Tabela 3) como na americana os percentuais foram similares, conforme a Tabela B6 (A) do manual (Wechsler, 1991). 
No WAIS-III, a amostra de padronização brasileira apresentou desempenho inferior ao da amostra americana quanto ao número máximo de dígitos memorizados nas ordens direta e inversa. A mediana do número máximo de dígitos memorizados pela amostra geral (Tabela 4) foi de cinco dígitos na ordem direta e quatro na inversa, enquanto na amostra geral americana a mediana foi de seis dígitos na ordem direta e cinco na inversa (Tabela B6 do manual do teste). Os desempenhos entre as faixas etárias variaram de um a dois dígitos a menos na retenção do número máximo de dígitos em ambas as ordens. Quanto à diferença entre o máximo de dígitos memorizados na ordem direta e inversa, os percentuais entre as amostras americana e brasileira foram diferentes (Wechsler, 1997). Observa-se que a diferença em favor da ordem inversa foi mais freqüente na amostra brasileira do que na americana.

As discrepâncias apontadas entre as pontuações brasileira e americana podem ser decorrentes de diferenças amostrais, tendo em vista, por exemplo, que as amostras americanas de normatização dos testes WISC-III e WAIS-III foram muito mais amplas do que as brasileiras. Também há que se considerar que essas discrepâncias em favor das amostras de países desenvolvidos têm sido igualmente identificadas em relação a diferentes habilidades cognitivas avaliadas por meio de testes de inteligência em estudos transculturais.

Tais resultados reforçam a necessidade de se realizar adaptações e padronizações de testes para novas populações, evitando o uso de referências para interpretações diferentes do padrão de desempenho da população para a qual o teste foi adaptado. As tabelas apresentadas contribuem para um exame clínico mais específico, permitindo analisar, separadamente, os diferentes processos cognitivos envolvidos nas duas tarefas do subteste Dígitos (Reynolds, 1997; Tulsky \& cols., 2003). Os procedimentos propostos por Kaplan compensam a limitação resultante da combinação dos escores brutos da ordem direta e inversa utilizada usualmente na interpretação do subteste, por ser a única possibilidade oferecida pelos manuais dos testes WISC-III e WAIS-III.

\section{Referências}

Cunha, J. A. (1993). As Escalas Wechsler. Em J. A. Cunha (Org.), Psicodiagnóstico-R. (pp. 278-354). Porto Alegre: Artes Médicas.

Eysenck, M. W. \& Keane, M. T. (1994). Psicologia Cognitiva: um manual introdutório. Porto Alegre: Artes Médicas.

Figueiredo, V. L. M. (2001). Uma adaptação brasileira do teste de inteligência WISC-III. Tese de Doutorado, Universidade de Brasília, Brasília.

Gregory, R. J. (1999). Foundations of intellectual assessment: the WAIS-III and other tests in clinical practice. Boston: Allyn and Bacon.
Glasser, A. J. \& Zimmerman, I. L. (1977). Interpretación clínica de la Escala de Inteligencia de Wechsler para Niños (WISC). Madrid: Tea.

Hale, J. B., Hoeppner, J. B. \& Fiorello, C. A (2002). Analyzing digit span components for assessment of attention processes. Journal of Psychoeducational Assessment, 20, 128-143.

Kaplan, E., Fein, D., Morris, R. \& Delis, D. C. (1991). WAIS-R as a Neuropsychological Instrument. San Antonio, TX: The Psychological Corporation.

Kaufman, A. S. (1990). Assessing adolescent and adult intelligence. Boston: Allyn.

Kaufman, A. I. (1994). Intelligent testing with the WISC-III. New York: John Wiley \& Sons, Inc.

Kaufman, A. S. \& Lichtenberger, E. O. (1999). Essentials of WAISIII Assessment. New York: John Wiley \& Sons, Inc.

Lezak, M. D. (1995). Neuropsychological assessment ( $3^{\mathrm{a}}$ ed.). New York: Oxford University Press.

Nascimento, E. (2000). Validação e adaptação do teste WAIS-III para um contexto brasileiro. Tese de Doutorado, Universidade de Brasília, Brasília.

Prifitera, A. \& Saklofske, D. (1998). WISC-III: Clinical use and interpretation. New York: Academic Press.

Reynolds, C. R. (1997). Forward and Backward Memory Span should not be combined for clinical analysis. Archives of Clinical Neuropsychology, 12(1), 29-40.

Rosenthal, E. N., Riccio, C. A, Gsanger, K. M. \& Jarratt, K. P. (2006). Digit Span components as predictors of attention problems and executive functioning in children. Archives of Clinical Neuropsychology, 21, 131-139.

Sattler, J. M. (1988). Assessment of children (3rd ed.). San Diego: Jerome M. Sattler, Publisher.

Sattler, J. M. \& Ryan, J. J. (1999). Assessment of children. Revised and updated third edition. WAIS-III supplement. San Diego: Jerome M. Sattler, Publisher.

The Psychological Corporation (1997). WAIS-III and WMS-III: technical manual. San Antonio: Autor.

Tulsky, D. S., Saklofske, D. H., Chelune, G. J., Heaton, R. K., Ivnik, R. J., Bornstein, R., Prifitera, A. \& Ledbetter, M. F. (2003). Clinical interpretation of the WAIS-III and WMS-III. New York: Academic Press.

Wechsler, D. (1991). Wechsler Intelligence Scale for Children -Third edition (WISC-III): Manual. San Antonio: Psychological Corporation.

Wechsler, D. (1997). WAIS-III: administration and scoring manual. San Antonio: Psychological Corporation.

Zimmerman, I. L. \& Woo-Sam, J. M. (1981). WAIS: Interpretación clínica de la Escala de Inteligencia de Wechsler para Adultos ( $3^{\mathrm{a}}$ ed.). Madrid: TEA Ediciones S.A.

Recebido em 13.11.2005

Primeira decisão editorial em 24.03.2006

Versão final em 26.09.2006

Aceito em 07.06.2007 